\title{
CHIR99021 combined with retinoic acid promotes the differentiation of primordial germ cells from human embryonic stem cells
}

\author{
Tingting Cheng ${ }^{1}$, Kui Zhai ${ }^{2}$, Yan Chang ${ }^{2}$, Guidong Yao ${ }^{1}$, Jiahuan He ${ }^{1}$, Fang Wang ${ }^{1}$, \\ Huijuan Kong ${ }^{1}$, Hang Xin ${ }^{1}$, Huiwen Wang ${ }^{2}$, Meng Jin ${ }^{2}$, Bing Gong ${ }^{3}$, Lei Gu ${ }^{2}$, Zhiguang \\ Yang ${ }^{2}$, Yanyun $\mathbf{W} \mathbf{u}^{2}$, Guangju $\mathrm{Ji}^{2}$, Yingpu Sun ${ }^{1}$ \\ ${ }^{1}$ Center for Reproductive Medicine, The First Affiliated Hospital of Zhengzhou University, Zhengzhou, China \\ ${ }^{2}$ National Laboratory of Biomacromolecules, Institute of Biophysics, Chinese Academy of Sciences, Beijing, China \\ ${ }^{3}$ Department of Cardiac Surgery, State Key Laboratory of Cardiovascular Disease, Fuwai Hospital, National Center for \\ Cardiovascular Disease, Chinese Academy of Medical Sciences and Peking Union Medical College, Beijing, China
}

Correspondence to: Guangju Ji, email: gj28@ibp.ac.cn

Yingpu Sun, email: syp2008@vip.sina.com

Keywords: CHIR99021, retinoic acid, primordial germ cells, human embryonic stem cells, $\beta$-catenin

Received: September 24, 2016

Accepted: December 01, 2016

Published: December 15, 2016

\section{ABSTRACT}

Primordial germ cells (PGCs) derived from human embryonic stem cells (hESCs) represent as a desirable experimental model as well as a potential strategy for treating male infertility. Here, we developed a simple and feasible method for differentiation of PGCs from hESCs by using CHIR99021 (an inhibitor of glycogen synthase kinase 3) and retinoic acid (RA). We firstly found that the deleted in azoospermia-like (DAZL) protein can be detected in 3 d CHIR99021 plus 9 d retinoic acid treated cultures and 12 d CHIR99021 plus retinoic acid co-treated cultures, but not expressed in single CHIR99021 treated cultures, single retinoic acid treated cultures, as well as $3 \mathrm{~d}$ retinoic acid plus $9 \mathrm{~d}$ CHIR99021 treated cultures. Next, we showed that several PGCs' markers were expressed in the $12 \mathrm{~d}$ CHIR99021 and retinoic acid co-treated cultures or $3 \mathrm{~d}$ CHIR99021 plus 9 d retinoic acid treated cultures. Moreover, meiosis was initiated in CHIR99021 and retinoic acid co-treated cultures as evidenced by a significant expression of the punctate synaptonemal complex protein 3 (SCP3). Fluorescent in situ hybridization (FISH) analysis indicated that a small percentage of putative $1 \mathrm{~N}$ populations were formed. Mechanically, we found that $\beta$-catenin relocated into nucleus after the treatment of $3 \mathrm{~d}$ CHIR99021 suggesting that Wnt signaling pathway was activated. Furthermore, blockade of Wnt signaling pathway by IWR-1 can reverse CHIR99021 and retinoic acid mediated-effects. Taken together, our results indicate that CHIR99021 combined with retinoic acid can effectively differentiate hESCs into PGCs via activating Wnt signaling pathway.

\section{INTRODUCTION}

Infertility affects approximately $10-15 \%$ of couples, with male factors accounting for $40-60 \%$ of all the cases. Although advances in assisted reproductive technologies (ART) have allowed these patients to have their offspring, the pathogenesis of infertility especially male infertility, such as non-obstructive azoospermia, immune caused azoospermia, and unexplained azoospermia are still not fully explored. Germ cells have been used for studying spermatogenesis in vitro and pathological mechanisms of male infertility. So far, much progress has been made in the derivation of male differentiated germ cells from hESCs and induced pluripotent stem cells (iPSCs) [1-17]. Moreover, several growth factors, such as bone morphogenetic protein 4 (BMP4), BMP7, BMP8b, stem cell factor (SCF), epidermal growth factor (EGF), retinoic acid (RA) have been used in the differentiation of PGClike cells (PGCLCs) from hESCs or iPSCs [2-6, 12, 15, $16,18]$. Besides, overexpression of the spermatogenesisrelated genes including DAZL and VASA (also called DDX4) seems to be an another strategy [9, 11]. It is reported that male gametes derived from mESCs/iPSCs function to produce the viable offsprings suggesting 
that there are possibilities to get germ cells in vitro from hESCs and to provide potential cures for male infertility. $[19,20]$. Thus, a comprehensive understanding of how to differentiate human male gametes is still urgent and necessary.

Spermatogenesis is an extremely complex process in vivo. It starts from spermatogonia to spermatozoa including a proliferative phase, a meiotic phase and a morphogenetic phase in most mammals [21]. Furthermore, the long-term production of billions of spermatozoa relies on the regulated proliferation and differentiation of spermatogonial stem cells (SSCs). The process of the transformation from SSCs into millions of haploid spermatozoa is elaborately organized in time and space [22].

Recently, the role of the Wnt/ $\beta$-catenin signaling pathway in spermatogenesis has been widely discussed both in mammalian and non-mammalian vertebrate species [23-26]. However, whether it is involved in the derivation of PGCs from hESCs in vitro is unclear. It is well known that activation of the canonical $\mathrm{Wnt} / \beta$ catenin signaling pathway by the Wnt ligand protein binds to Frizzled and Lrp5/6 receptors inhibits the GSK3 $\beta$ (glycogen synthase kinase $3 \beta$ )-mediated degradation of $\beta$-catenin and results in the accumulation of cytoplasmic $\beta$-catenin. Then it translocates into the nucleus where it interacts with LEF/TCF transcriptional complexes to regulate the downstream target genes [27-29]. Here, we describe a crosstalk between CHIR99021 and RA in the differentiation of PGCs from hESCs via activating Wnt signaling pathway. CHIR99021 activated Wnt signaling pathway and then initiated the differentiation of hESCs by inhibiting the GSK $3 \beta$ mediated degradation of $\beta$-catenin; meanwhile, RA upregulated c-Kit. Those two compounds together promote the emergence of PGCs from hESCs. Our results highlight a simple and flexible method for inducing PGCs from hESCs that may provide a broad implication for studying male infertility.

\section{RESULTS}

\section{CHIR99021 combined with RA induces the emergence of primitive DAZL-positive cells}

To access whether CHIR99021 and/or RA can induce PGCs from hESCs (Figure 1A), DAZL protein was initially detected by using immunofluorescence experiments. As shown in Figure 1B, among the different treatments, single CHIR99021, single RA and 3 d RA plus 9 d CHIR99021 did not induce the emergence of DAZL-positive cells. In contrast, DAZL protein was found to be expressed in cells treated by $3 \mathrm{~d}$ CHIR99021 plus 9 d RA and 12 d CHIR99021 plus RA (co-culture) groups. These DAZL-positive cells accounted for nearly $8-10 \%$ of the total cells. In addition, there were more DAZL-positive cells in the $12 \mathrm{~d}$ CHIR99021 plus RA (co-culture) treatment compared to the 3 d CHIR99021 plus 9 d RA treatment but without any significant difference (as shown in Figure 1C). Collectively, these results illustrate that CHIR99021 and RA can work together to induce PGCs from hESCs.

\section{DAZL-positive cells exhibit a phenotype comparable to that of migratory PGCs}

To explore the phenotype of these induced cells, several markers of PGCs such as DDX4, Blimp-1, Nanos, and TFAP2C were measured in our experimental conditions. As shown in Figure 2A, we found that the mRNA levels of several PGC markers including DDX4, Blimp-1, Nanos and TFAP2C were significantly upregulated in the $3 \mathrm{~d}$ CHIR99021 plus $9 \mathrm{~d}$ RA and $12 \mathrm{~d}$ CHIR99021 plus RA (co-culture) groups. Besides, in contrast to the undifferentiated hESCs, the expression levels of acrosin and DDX4 were significantly higher in both lines. Furthermore, $\beta$-catenin had a significantly increased expression compared with the undifferentiated group (Figure 2B).

Flow cytometry analysis was implemented to further determine the phenotype of these induced cells. c-Kit, a critical cell surface marker that distinguishes differentiated spermatogonia (also called Kit), together with RA, can direct the differentiation of spermatogonia throughout the male reproductive lifespan [30]. We observed the frequencies of c-Kit positive cells in the $3 \mathrm{~d}$ CHIR99021 plus $9 \mathrm{~d}$ RA and $12 \mathrm{~d}$ CHIR99021 plus RA (co-culture) groups were $26.9 \%$ and $24 \%$, respectively (Figure 2C). Here, we used the chemokine receptor CXCR4 as a positive marker, given that $\mathrm{CXCR} 4$ receptor is mainly expressed on the migrating germ cells [31], we found that CXCR4 positive cells account for approximately $13.2 \%$ in 12 d CHIR99021 plus RA (co-culture) group. However, the percentage of CXCR4 positive cells in $3 \mathrm{~d}$ CHIR99021 plus $9 \mathrm{~d}$ RA are lower than the co-culture group and just account for $2.95 \%$ which may explained as migrating germ cells was less in this induced process(Figure 2D). Furthermore, the percentage of DAZL positive cells in the $3 \mathrm{~d}$ CHIR99021 plus $9 \mathrm{~d}$ RA and $12 \mathrm{~d}$ CHIR99021 plus RA (co-culture) groups were $24.7 \%$ and $41.1 \%$, respectively (Figure $2 \mathrm{E}$ ). Taken together, these results further confirmed that CHIR99021 combined with RA can effectively differentiate hESCs into PGCs.

\section{Putative haploid cells were formed in CHIR99021 and RA treated cultures}

Previous studies showed that post-meiotic germ cells can be obtained from hESCs $[1,11,32]$. We next determined whether meiotic was happened and even haploid cells were produced. Here, we used SCP3 which is indicative biomarker of synaptonemal complex formation in meiotic prophase I to examine the meiotic progress in our experiments [33]. As shown in Figure 3A, compared 
Table 1: List of Q-PCR primer sequences used in this study (Related to experimental procedures)

\begin{tabular}{lcc}
\hline \multicolumn{1}{c}{ Genes } & \multicolumn{1}{c}{ Forward } & Reverse \\
\hline DDX4 & CCATTGTGGATGTATTATCTCGCTTA & GCCTCTGGGCGGAATTTT \\
Blimp-1 & AACTTCTTGTGTGGTATTGTCGG & CAGTGCTCGGTTGCTTTAGAC \\
Nanos & GACGCTTCTGCCCACTTACTG & GTCCTGTGTCTTCGCCTTGTC \\
TFAP2C & TCAGTCCCTGGAAGATTGTCG & CCAGTAACGAGGCATTTAAGCA \\
GAPDH & GAAATCCCATCACCATCTTCCAGG & GAGCCCCAGCCTTCTCCATG \\
\hline
\end{tabular}

A

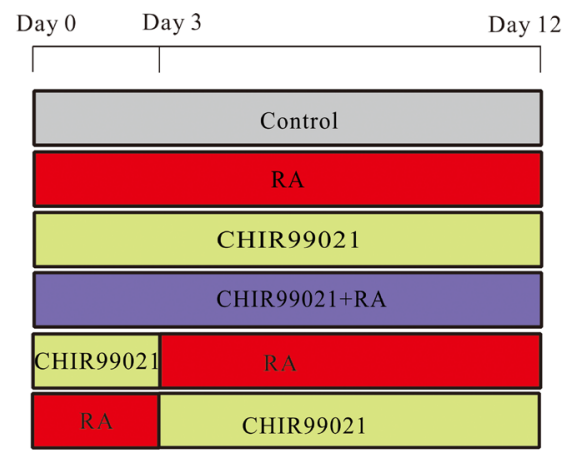

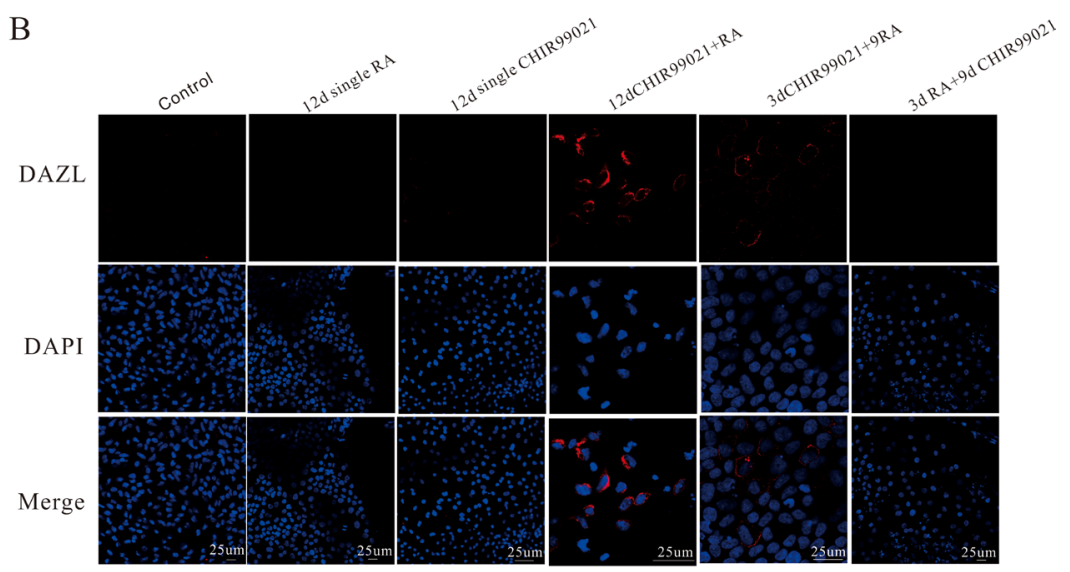

C

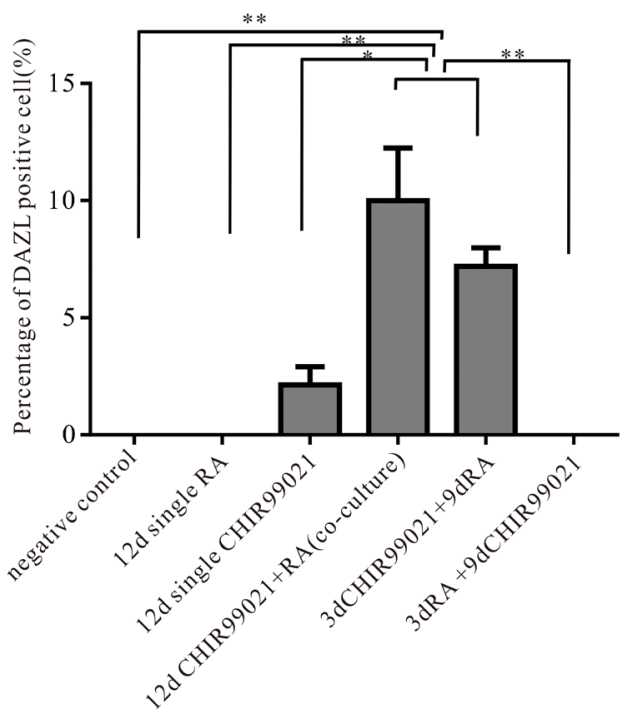

Figure 1: CHIR99021 combined with RA can induce the PGCs from hESCs. (A) The protocol used for the differentiation of hESCs into PGCs. (B) The hESCs were treated by CHIR99021 and/or RA as indicated in (A). The DAZL positive cells were detected by using immunofluorescence staining nuclei were counterstained with DAPI. Scale bars, $25 \mu \mathrm{m}$. (C) Summary data showing the mean number of the DAZL positive cells in the different treatments. All data are presented as means \pm SEM. $* p<0.05, * * p<0.01$. 

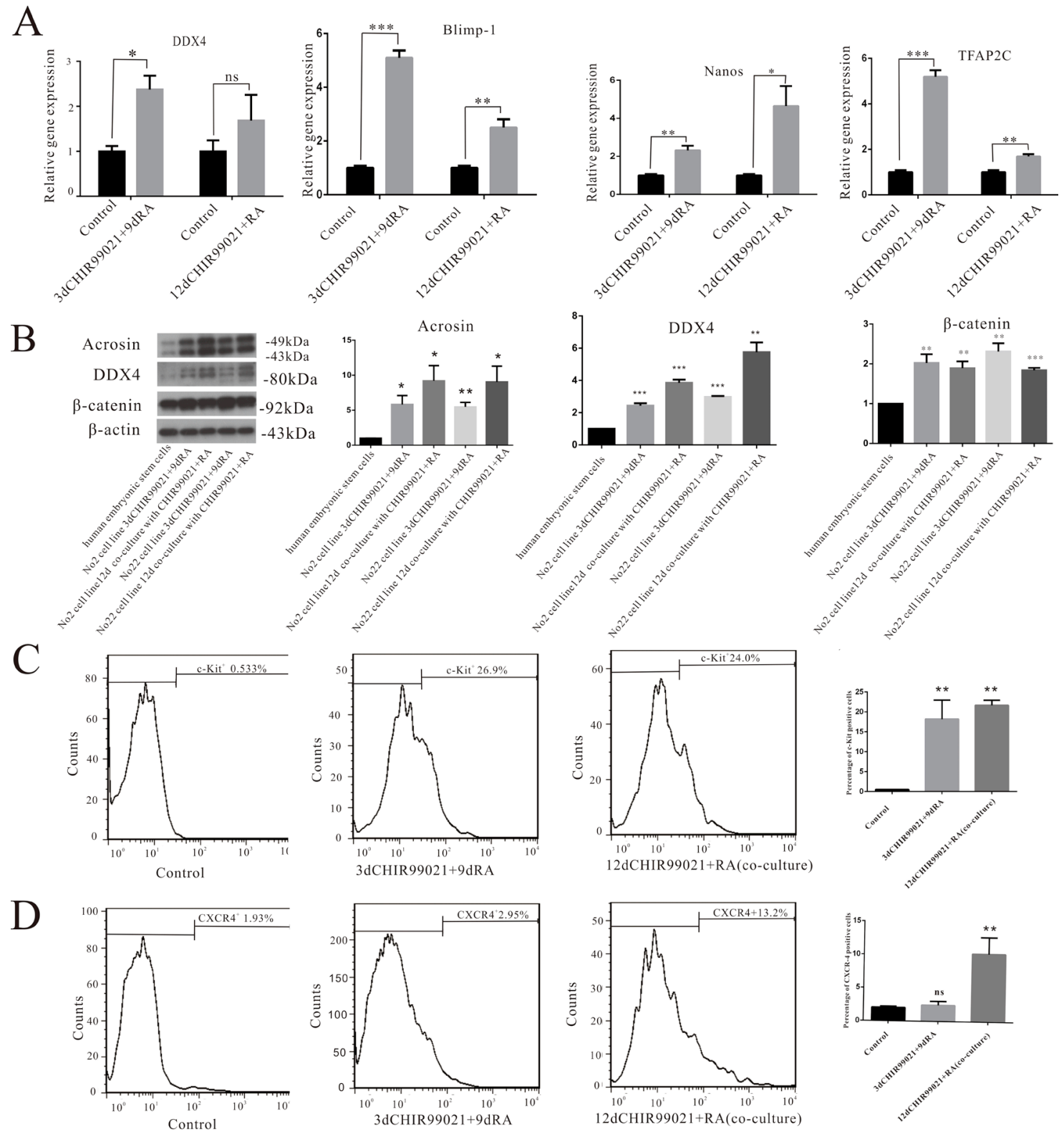

$\mathrm{E}$
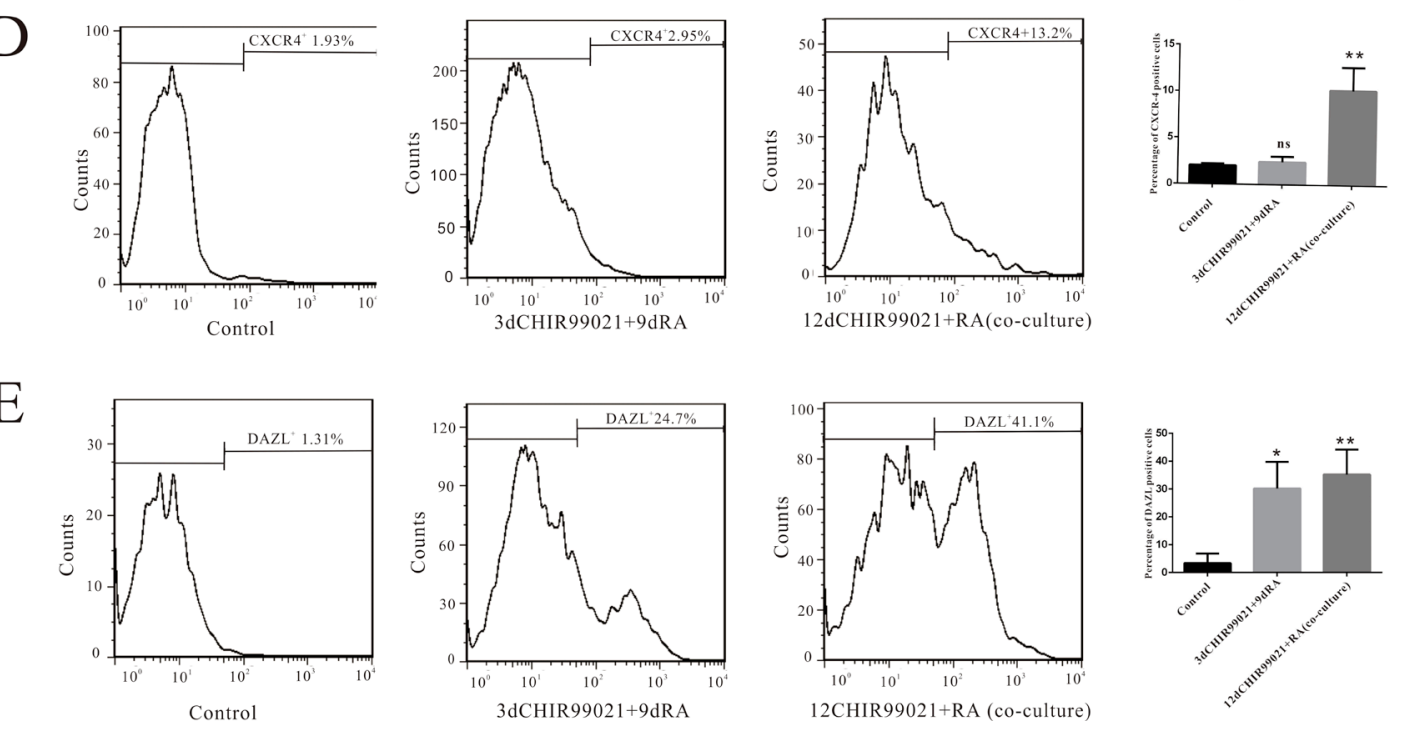

Figure 2: DAZL-positive cells have a phenotype comparable to that of migretory PGCs. (A) mRNA levels of DDX4, Blimp-1, Nanos and TFAP2C was determined by RT-qPCR. The two hESCs lines were treated by $12 \mathrm{~d}$ CHIR99021 plus RA co-culture and $3 \mathrm{~d}$ CHIR99021 plus $9 \mathrm{~d}$ RA. The undifferentiated hESCs were used as control. (B) The two hESCs lines were treated by $12 \mathrm{~d}$ CHIR99021 plus RA co-culture or $3 \mathrm{~d}$ CHIR99021 plus $9 \mathrm{~d}$ RA. The undifferentiated hESCs were used as control. Then, Acrosin, DDX4, $\beta$-catenin and $\beta$-actin were detected by Immunoblots. All data are presented as means \pm SEM in three independent experiments vs undifferentiated hESCs. (C-E) Flow cytometry analysis of c-Kit ${ }^{+}, \mathrm{CXCR}^{+}$and DAZL ${ }^{+}$cells. The undifferentiated hESCs were used as a control shown on the left. The percentage shown in the histogram is the rate of positive cells. The middle icon represents $3 \mathrm{~d}$ CHIR99021 plus $9 \mathrm{~d}$ RA, the right icon stands for $12 \mathrm{~d}$ CHIR99021 and RA co-culture treatment. All data are presented as means \pm SEM in three independent experiments. ${ }^{*} p<$ $0.05, * * p<0.01, * * * p<0.001$. 
with undifferentiated hESCs group, punctate pattern was significantly increased in both induced groups indicating that the initiation of meiosis starts. Furthermore, FISH analysis was carried out to verify whether haploid cells were produced. As shown in Figure 3B, two probes for chromosome 16 and 22 were used. As expected, putative $1 \mathrm{~N}$ cells possessed a single chromosome 16 and 22, whereas undifferentiated hESCs (2N) carried 2 chromosomes. These results indicate that human primordial germ cells differentiated from hESCs could even possess the ability to enter into the meiosis and form the haploid cells.

\section{Wnt signaling pathway was involved in the initiation of the differentiation of hESCs into PGCs}

It is reported that long time activation of $\mathrm{Wnt}$ signaling pathway can induce the differentiation of hESCs [34]. Consistent with this study, we treated on the hESCs with CHIR99021 for $3 \mathrm{~d}$. Compared to the undifferentiated hESCs, the immunofluorescence results (Figure 4A) clearly showed that increased $\beta$-catenin had been obviously translocated from the cytoplasm into the nucleus. In addition, these percentage of $\beta$-catenin accounted for 40\% (Figure 4B).

IWR-1 is known to suppress the canonical Wnt signaling pathway through stabilizing the Axin and the $\beta$-catenin destruction complex [35]. To verify the role of Wnt signaling pathway in CHIR99021 mediated effects, we added IWR-1 into the 3 d CHIR99021 treatment and CHIR99021 combined with RA co-culture. As shown in Figure 5A, compared with the undifferentiated hESCs, 3 d CHIR99021 and IWR-1 co-culture treatment not only markedly reduced expression of Oct 4 , but also prevented $\beta$-catenin from translocating into the nucleus. Therefore, the downstream of Wnt signaling pathway cannot be initiated.

Compared to the CHIR99021 and RA co-culture treatment, the DAZL protein was not induced in the CHIR99021, IWR-1 and RA co-culture group (Figure 5B) illustrating that Wnt signaling pathway plays a very important role in inducing PGCs. These results further prove our hypothesis that the activation of the Wnt signaling pathway by CHIR99021 plays a decisive role in the whole process.

\section{DISCUSSION}

In this study, we showed that CHIR99021 combined with RA induced PGCs from hESCs. At the beginning, the $12 \mathrm{~d}$ CHIR99021 and RA co-culture and the $3 \mathrm{~d}$ CHIR99021 plus 9 d RA groups were found to promote the expression of DAZL, which is a germ cell specific RNA binding protein and plays an indispensible role in germ cell development in mammals both in vivo and in vitro $[11,32,36,37]$. Subsequently, western blotting, the RT-qPCR and the flow cytometry provided strong evidence to support the fact that these two small molecules do have an effect on the induction of PGCs. SCP3 straining and FISH analysis confirmed that meiosis was initiated and putative haploid cells were produced. From a perspective of reproductive medicine, we provide a simple and feasible method for generating PGCs in vitro and solve the practical and ethical difficulties associated with obtaining human tissue in early development. This method may serve as an important tool for further exploring the development of germ cells in vitro and deciphering the pathological mechanisms of male infertility.

RA, a metabolite of vitamin A (retinol), mediates the functions of vitamin $\mathrm{A}$ and is required for growth and development. There is a general consensus that dietary retinol or vitamin A is needed for normal spermatogenesis [38]. Recently, researchers have found that RA can not only trigger spermatogonial differentiation through Oct4 and PLZF [39], but also play an indispensible role in promoting the translation of the Kit mRNAs via activating the PI3K/ AKT/mTOR signaling pathway [30]. As an important inducible factor, RA was reported to participate in inducing the embryoid body (EB) derived from human/mouse embryonic stem cells to differentiate toward germ cells with or without other exogenous factors in vivo [40-43]. However, we used directed differentiation system with $\mathrm{RA}$, so it may explain why single RA-treatment on hESCs differentiation could not induce PGCLCs when this culture system was used.

As described above, CHIR99021 can activate the Wnt signaling pathway and promote free cytoplasmic $\beta$-catenin to accumulate and even exceed the binding capacity of E-cadherin, leading to the translocation of $\beta$-catenin into the nucleus and the regulation of the downstream LEF/TCF transcriptional complexes [27-29, 34]. Meanwhile, much progress has been reported recently about the role of CHIR99021 in inducing hESCs/iPSCs into different cell types, including cells expressing makers of the mesendoderm, the nephrogenic intermediate mesoderm and cardiomyocytes [44-47]. Remarkably, a recent genome-wide expression analysis showed that 6-bromoindirubin-3'-oxime (BIO, another GSK3 inhibitor) can significantly upregulate the reproductive development process, gland development and the development of primary sexual characteristics in mESCs [48]. However, the role of CHIR99021 in inducing hESCs into PGCs is much less known. Here, we found that CHIR99021 combined with RA can promote hESCs direct differentiation into PGCs. Similar to the previous studies, our results showed that the Wnt signaling pathway was also activated by a $3 \mathrm{~d}$ treatment with GSK3 inhibitor, along with $\beta$-catenin being noticeably translocated into the nucleus.

To further explore the contribution of CHIR99021 to differentiation, we chose a small-molecule inhibitor 

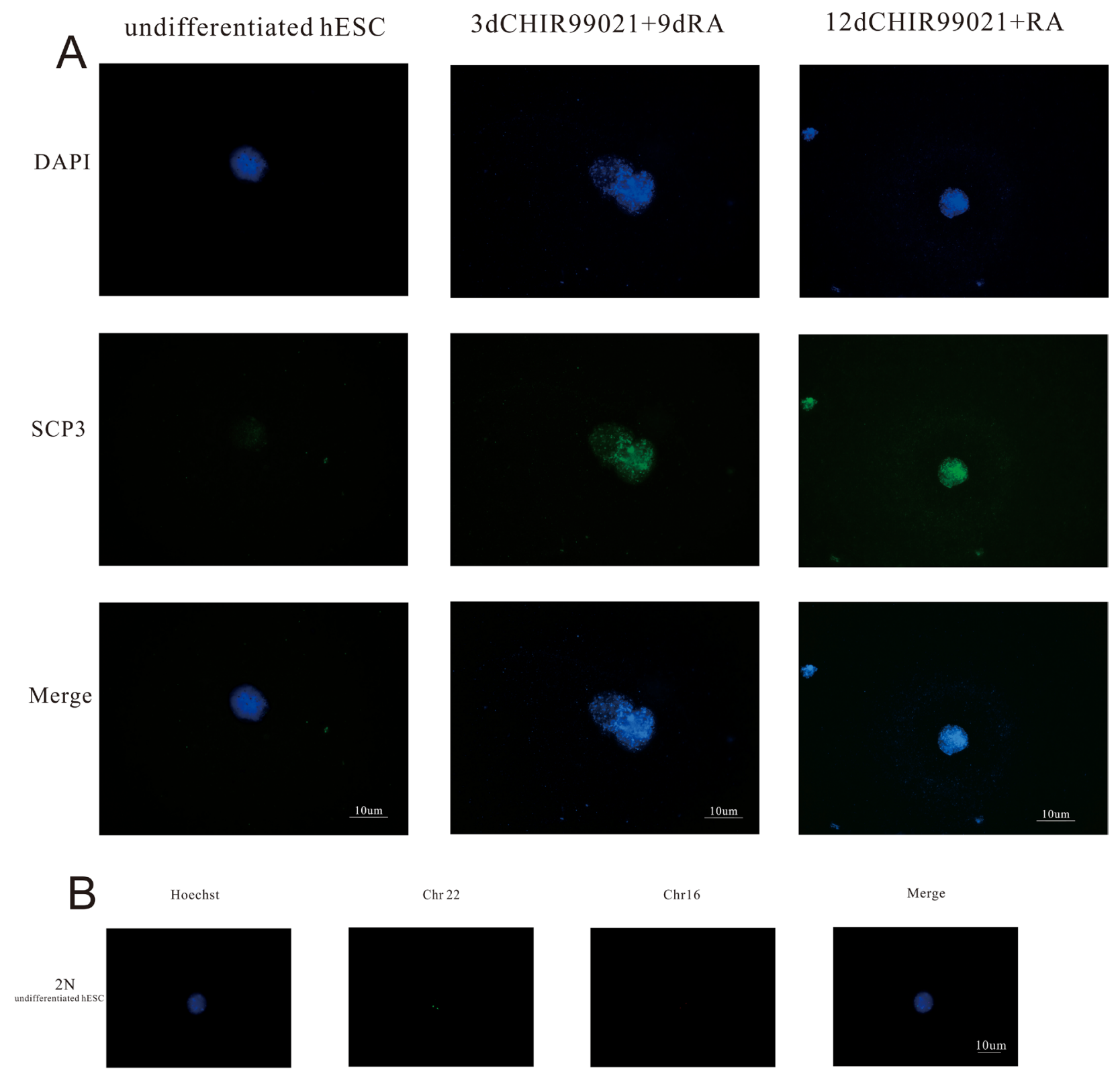

Chr 22

Chr16
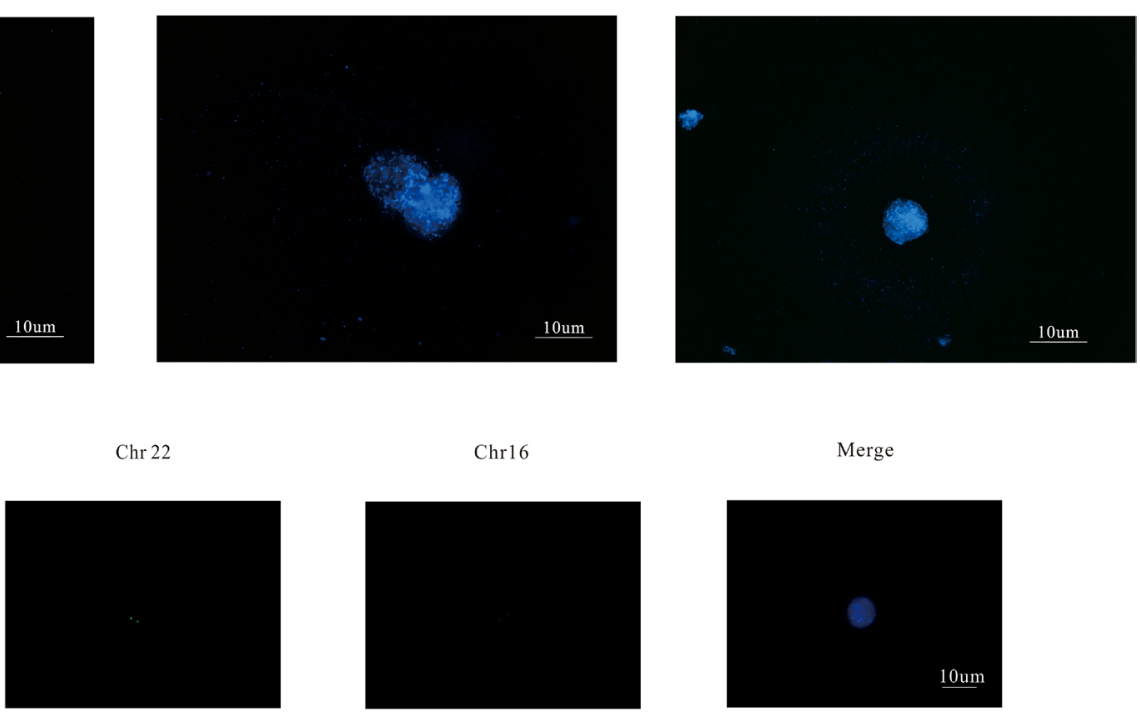

Merge
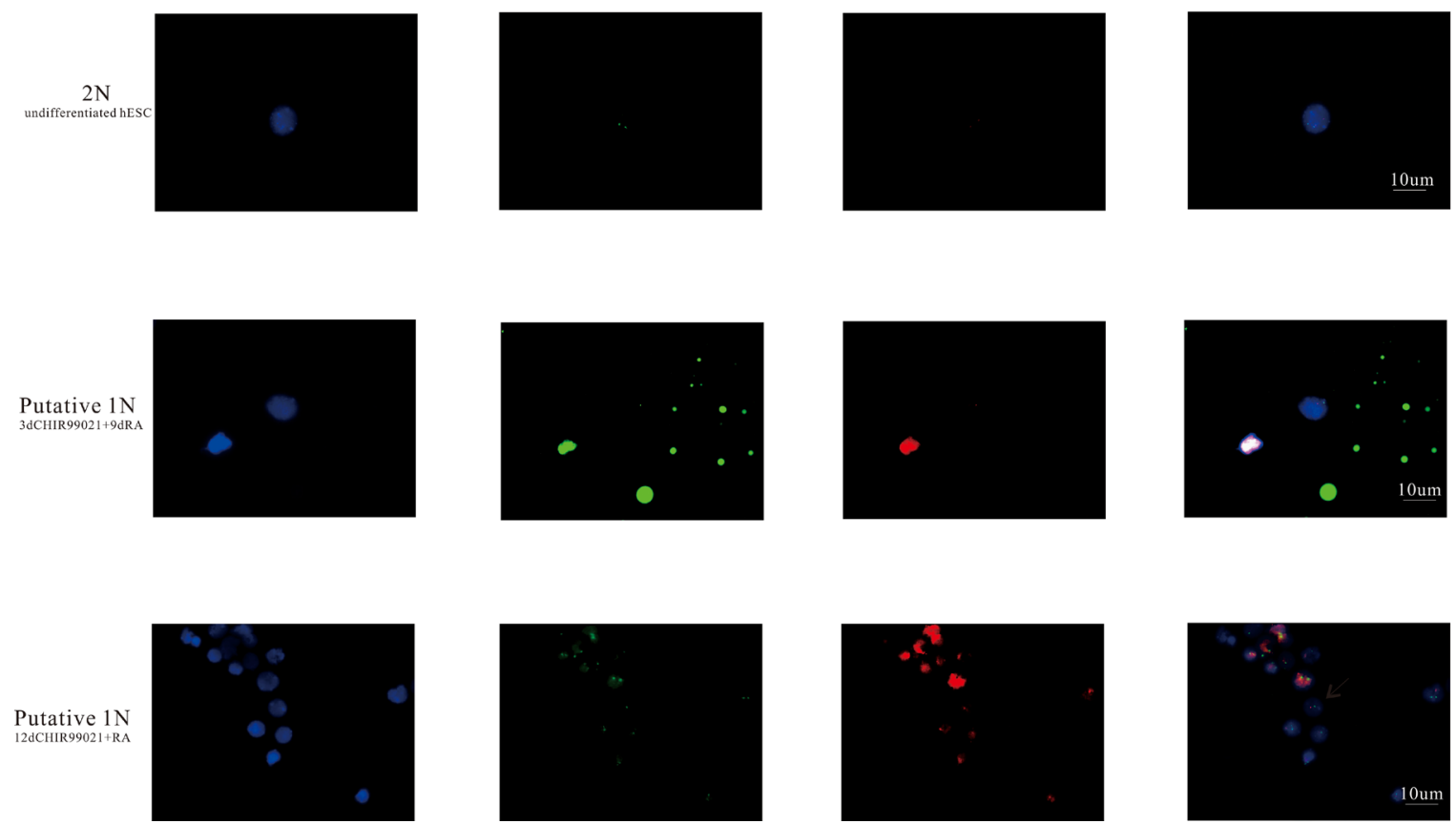

Figure 3: Putative haploid cells were formed in this induced process. (A) Immunofluorescence staining with SCP3 is shown. Scale bars, $10 \mu \mathrm{m}$. (B) FISH with probe against autosomal chromosome 16 and chromosome 22 in the undifferentiated hESCs group and two induced groups. Scale bars, $10 \mu \mathrm{m}$. 
of the Wnt signaling pathway, IWR-1. It induces Axin2 protein levels and promotes $\beta$-catenin phosphorylation by stabilizing Axin-scaffolded destruction complexes. After blockade the Wnt signaling pathway, we can not observe that the translocation of $\beta$-catenin into nucleus and the expression of DAZL protein in the optimum induced protocol. Thus, we recognized that the 3 d CHIR99021 following $9 \mathrm{~d}$ RA may work as follows: the activated Wnt signaling pathway by CHIR99021 firstly causes the translocation of $\beta$-catenin into the nucleus; then, RA induces the emergence of PGCs by the up-regulation of c-Kit. Moreover, the co-culture with CHIR99021 and RA may explain that the Wnt signaling pathway was constitutively activated by CHIR99021 even past the first $3 \mathrm{~d}$ (during the remaining $9 \mathrm{~d}$ ), and RA acts in the same function as described above (Figure 6). In the future, it would be very interesting to test how to induce mature sperm cells and to improve the efficiency of induced sperm cells.
In summary, we provide a simple and feasible method for the induction of hESCs to differentiate into PGCs. In brief, CHIR99021 promoted the $\beta$-catenin translocating into the nucleus and activated the Wnt signaling pathway. Then, RA induced the expression of c-Kit and further induced the hESCs to differentiate into PGCs. In the future, "the PGCs" induced by our method may be used as a model for the screening of drugs and studying of male infertility related disease.

\section{MATERIALS AND METHODS}

\section{hESCs lines and culture media}

The human ESC lines Zh2 and Zh22 (ZZU-hESC-2 and ZZU-hESC-22, ZZU: Zhengzhou University) were obtained from the University of Zhengzhou. Both lines were established by isolating the inner cell mass of

A

$\beta$-catenin

Oct4

DAPI

Merge

Control
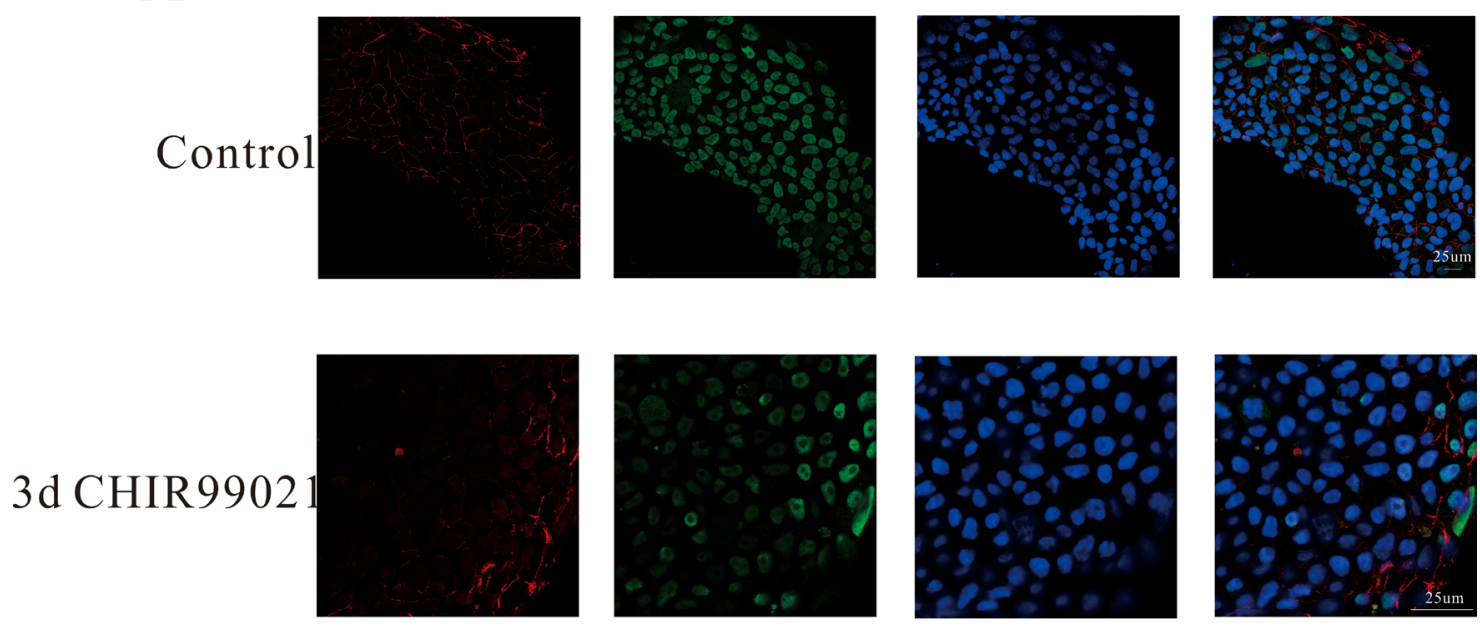

B

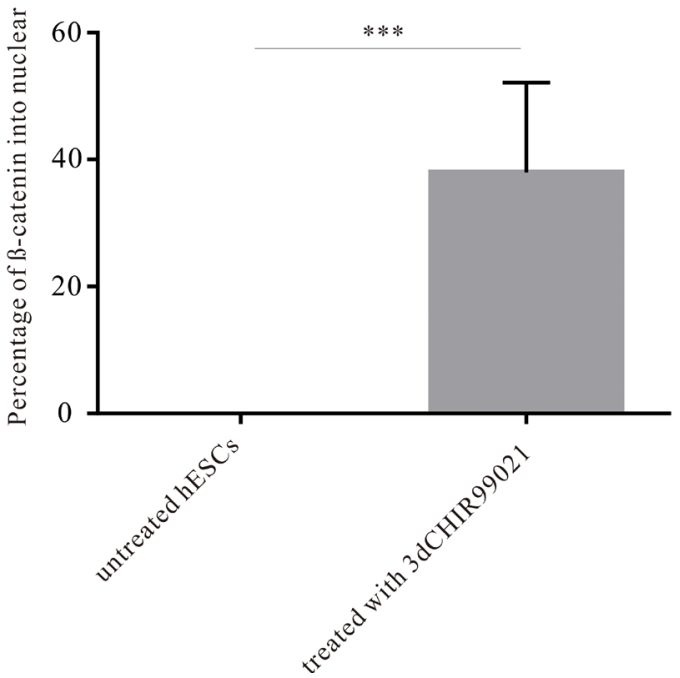

Figure 4: CHIR99021 activated Wnt signaling pathway via a long-term manner. (A) Immunofluorescence analysis showing the expression of OCT4, DAZL, and $\beta$-catenin after the treatment of $3 \mathrm{~d}$ CHIR99021. Scale bars, $25 \mu \mathrm{m}$. (B) Statistical analysis of the percentage of $\beta$-catenin in the nuclei. All data are presented as means \pm SEM. ${ }^{*} p<0.05,{ }^{* *} p<0.01,{ }^{* * *} p<0.001$. 
in vitro fertilized $3 \mathrm{AA}$ and $5 \mathrm{AA}$ blastocysts provided by Embryonic Stem Cell Laboratory of the Reproductive Medical Center of the First Affiliated Hospital of Zhengzhou University as described previously [49]. Both lines had male Karyotypes with 46, XY. The two hESCs lines were routinely grown in $35 \mathrm{~mm}$ dishes on inactivated primary mouse embryo fibroblasts (PMEF) and cultured by a hESCs growth medium consisting of Dulbecco's modified Eagle's medium (DMEM)-Ham's F-12 medium (F12) supplemented with 20\% Knockout serum replacement (KSR), 1\% nonessential amino acids (NEAA), 2 mM L-glutamine, $0.1 \mathrm{mM} \beta$-mercaptoethanol, and $10 \mathrm{ng} / \mathrm{ml}$ of basic fibroblast growth factor (all from GIBCO, USA). In order to induce the PGCs, hESCs were transferred into feeder-free conditions with conditioned medium obtained by incubating culture medium with MEFs for $24 \mathrm{~h}$ supplemented with $10 \mathrm{ng} / \mathrm{ml} \mathrm{bFGF}$. After passaging for 2 generations, the hESCs were divided into 5 groups and treated as follows: i: single $8 \mu \mathrm{M}$ CHIR99021 for $12 \mathrm{~d}$; ii: single $2 \mu \mathrm{M}$ RA for $12 \mathrm{~d}$; iii: combined with both $8 \mu \mathrm{M}$ CHIR99021 and $2 \mu \mathrm{M}$ RA for $12 \mathrm{~d}$; iv: $8 \mu \mathrm{M}$ CHIR99021 was added in the first three days, and then single $2 \mu \mathrm{M}$ RA was used for the next $9 \mathrm{~d}$; v: adding $2 \mu \mathrm{M}$ RA for the first three days and then single $8 \mu \mathrm{M}$ CHIR99021 for the next $9 \mathrm{~d}$. Both CHIR99021 and RA mentioned above were diluted in hESCs medium without bFGF (see Figure 1A). Moreover, $5 \mu \mathrm{M}$ IWR-1 was used in this study.

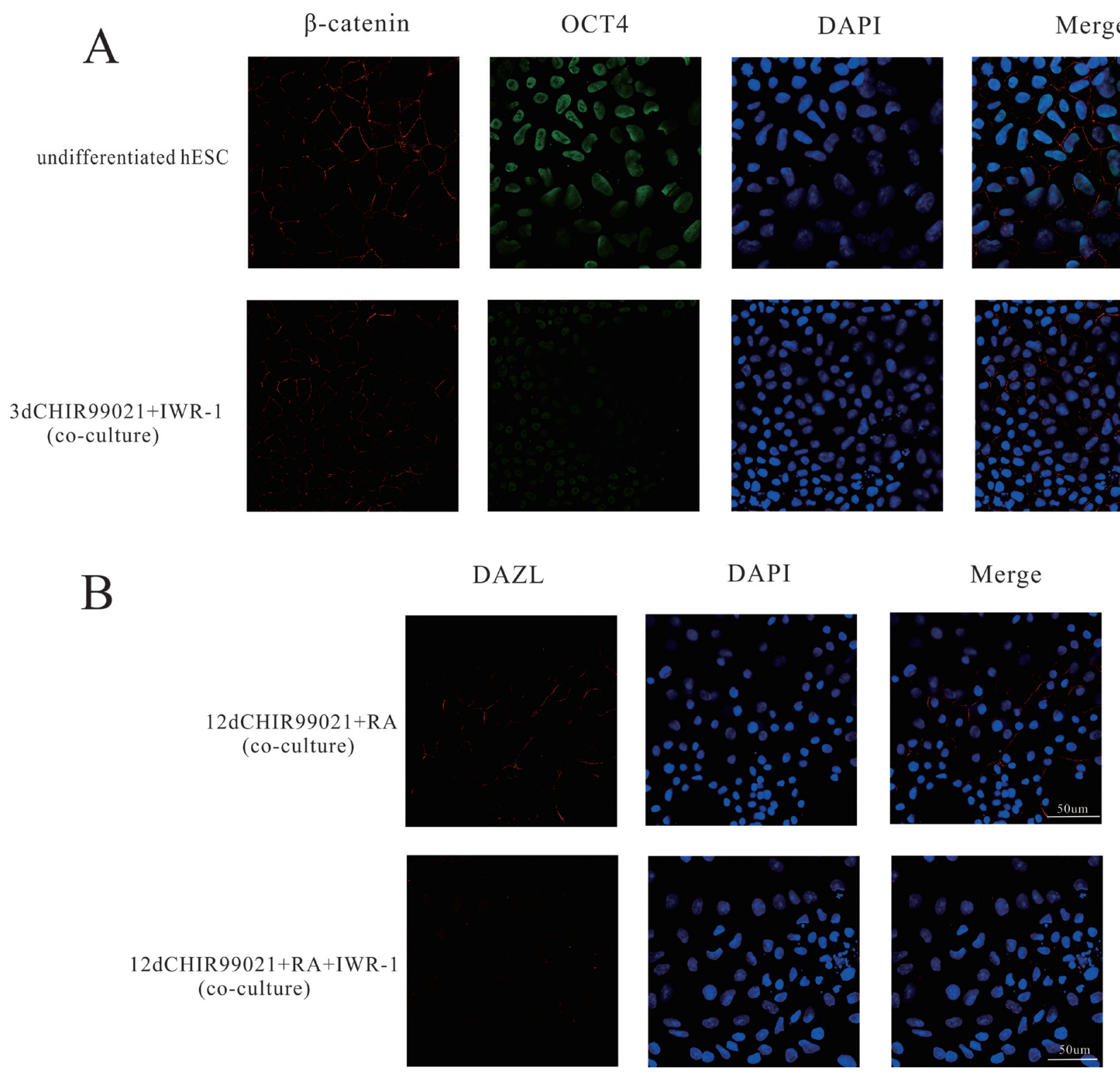

Figure 5: IWR-1 inhibited the accumulation of $\beta$-catenin in the nuclei and decreased the number of DAZL positive cells. (A) Immunofluorescence straining of $\beta$-catenin and OCT4 in 3 d CHIR99021 plus IWR-1 co-culture group and undifferentiated group. Scale bars, $50 \mu \mathrm{m}$. (B) Immunofluorescence straining of DAZL in 12 d CHIR99021, IWR-1 and RA co-culture group and undifferentiated group. Scale bars, $50 \mu \mathrm{m}$. 


\section{Immunofluorescence}

The cells were washed twice with phosphate buffer solution (PBS) and then fixed with 4\% paraformaldehyde for $15 \mathrm{~min}$ at room temperature. After washing twice with PBS, the cells were subsequently permeabilized with $0.1 \%$ triton X-100 for 10 min and then blocked with $5 \%$ bovine serum albumin (BSA) for $30 \mathrm{~min}$. After the blockade, the cells were stained with the following antibodies: anti-DAZL (ab34139; abcam), anti-Oct4 (MAB4419A4; Millipore) and anti- $\beta$-catenin (\#9562; Cell Signaling Technology) overnight at $4^{\circ} \mathrm{C}$. After washing twice with PBS and incubating with 5\% BSA for $30 \mathrm{~min}$, the cells were incubated with the second antibodies (Alexa Fluor 488-conjugated goat anti-mouse IgG, Alexa Fluor 574-conjugated goat anti-rabbit $\operatorname{IgG}$, Invitrogen) for $1 \mathrm{~h}$ at room temperature. After washing, the cells were incubated in diamibino-phenyl-indole (DAPI, 14016-01, BIODEE) for $30 \mathrm{~min}$. Finally, the cells were mounted on slides and examined by using a laser scanning confocal microscope (Leica SP5). The images were analyzed by using the FIJI software.

\section{Western blotting}

After rinsing with cold PBS, the cells were harvested and lysed on ice with RIPA buffer containing phenylmethanesulfonyl fluoride for $30 \mathrm{~min}$. Then, the liquid was transferred into $1.5 \mathrm{~mL}$ tubes and centrifuged at $12,000 \mathrm{~g}$ for $25 \mathrm{~min}$ to obtain the supernatant. The protein concentrations were determined by using the $\mathrm{BCA}$ assay. Before loading on gels, the cell lysates were heated at $90^{\circ} \mathrm{C}$ for $5 \mathrm{~min}$ in $2 \mathrm{X}$ sample loading buffer containing $5 \% \beta$-mercaptoethanol. Five $\mathrm{mg}$ proteins were resolved on a $10 \%$ SDS gel. The resolved proteins were transferred to PVDF membrane (Millipore) at $300 \mathrm{~mA}$ for $1.5 \mathrm{~h}$. The membranes were blocked for $1 \mathrm{~h}$ with Tris-buffered saline-Tween 20 (TBST) containing 5\% skim milk powder

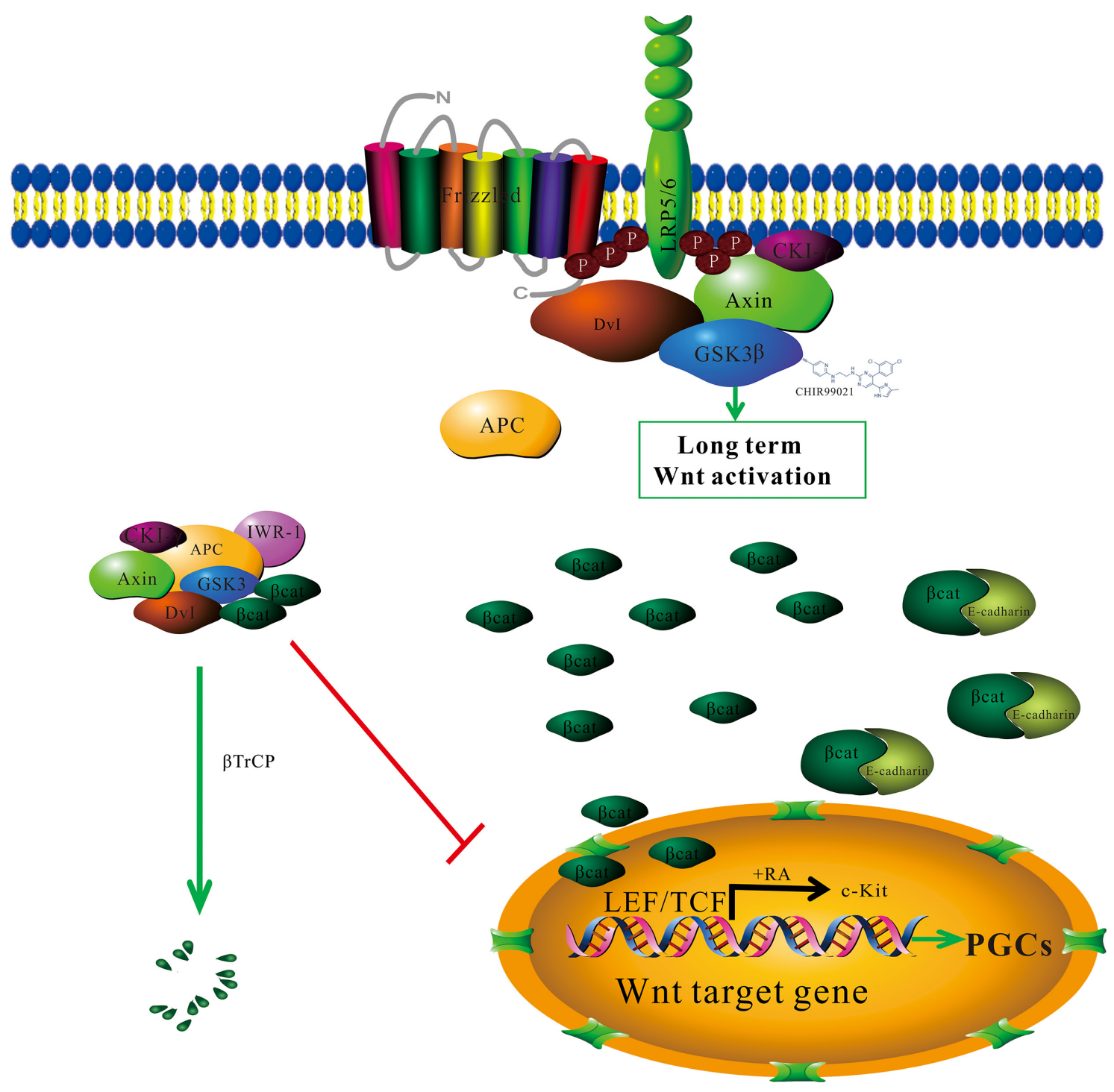

Figure 6: The proposed working model. 
at room temperature. Then, the PVDF membranes were incubated overnight with anti-DDX4 (\#8827; 1:1000, Cell Signaling Technology), anti- $\beta$-catenin (\#9562; 1:1000, Cell Signaling Technology), anti-acrosin (sc-46284; 1:200, Santa Cruz Biotechnology) and anti- $\beta$-actin (60008-1-AP; 1:20000, Proteintech) at $4^{\circ} \mathrm{C}$. After washing and incubating with the secondary antibodies, the final detection was performed using enhanced chemiluminescence detection solutions 1 and 2 (1:1) (ECL, Millipore). The proteinsignal densities were normalized to the corresponding $\beta$-actin-signaling densities.

\section{Fluorescent in situ hybridization}

For FISH analysis, the cells were harvested and dissociated into single cells with $0.25 \%$ trypsin and then washed in PBS. Cell pellets were treated with hypotonic solution $(0.075 \mathrm{M} \mathrm{KCL})$ for $25 \mathrm{~min}$ at $37^{\circ} \mathrm{C}$, then fixed twice with Carnoy's fixative (1:3 acetic acid: methanol) for $20 \mathrm{~min}$ and then cell pellets were dropped onto the slides and dried at $56^{\circ} \mathrm{C}$ in drying box for $2 \mathrm{~h}$. Then the slides were washed (Sangon, Shanghai, China) in 2X SSC for $10 \mathrm{~min}$, following by a dehydrated procedure in $80 \%, 90 \%$ and $100 \%$ ethanol series for 3 min each. FISH probes (Chr16, Chr 22, GP, China) were denatured on slides at $76^{\circ} \mathrm{C}$ for 6 min on a hot plate and further hybridized at $42^{\circ} \mathrm{C}$ in a moist chamber for $16 \mathrm{~h}$. In the following step, the slides were washed with $0.3 \% \mathrm{NP}$ $40 / 0.4 \mathrm{X} \mathrm{SSC}$ for $2 \mathrm{~min}, 0.1 \% \mathrm{NP}-40 / 2 \mathrm{X} \mathrm{SSC}$ for $30 \mathrm{sec}$ and $70 \%$ ethanol for $3 \mathrm{~min}$, respectively. Finally, the slides were strained with DAPI.

\section{Immunofluorescence staining of synaptonemal complexes}

For the straining of synaptonemal complexes, cells were lysed by a hypotonic solution for $30 \mathrm{~min}$ at room temperature, following submerged in 1\% PFA on glass slides overnight at room temperature. The slides were washed with $\mathrm{ddH}_{2} \mathrm{O}$ for $4 \mathrm{~min}$, then blocked with ADB buffer (diluting $1 \%$ donkey serum, $0.3 \%$ BSA and $0.01 \%$ triton $\mathrm{X}-100$ in PBS buffer) at room temperature for $30 \mathrm{~min}$ and incubated with primary antibody against human SCP3 (mouse polyclonal, 1:200, Abcam) overnight at $37^{\circ} \mathrm{C}$. The slides were further washed with $1 \mathrm{X}$ TBS $10 \mathrm{~min} 3$ times following blocked with ADB buffer for $6 \mathrm{~h}$ at $4^{\circ} \mathrm{C}$, and then washed with $1 \mathrm{X}$ TBS 10 min 3 times again and incubated with goat anti-mouse FITC-conjugated (1:120, Santa) secondary antibodies at $37^{\circ} \mathrm{C}$ for $1.5 \mathrm{~h}$ and mounted as described above.

\section{Real-time RT-qPCR}

Total RNA was extracted from the cells with the TRIZOL RNA purification system (Invitrogen). According to the manufacturer's instructions (TAKARA), cDNA was generated from the mRNA $(1 \mu \mathrm{g})$ using the PrimeScript ${ }^{\mathrm{TM}}$
RT reagent Kit with gDNA Eraser (RR047A, TAKARA), Quantitative real-time RT-qPCR was performed using EvaGreen 2X qPCR Mix (master Mix-S, abm) in a RoterGene Q-QIAGEN machine RT-PCR with the following profile: $95^{\circ} \mathrm{C}$ for $10 \mathrm{~min}$, followed by 40 cycles of $95^{\circ} \mathrm{C}$ for $15 \mathrm{~s}$ and $60^{\circ} \mathrm{C}$ for $60 \mathrm{~s}$. The amount of Evagreen signal was recorded at the end of each cycle. The levels of target mRNAs analyzed by RT-qPCR were normalized to the level of GAPDH. Detailed information regarding the primer pairs used in this experiment was shown in Table 1. The data presented are the average of at least 3 independent experiments.

\section{Flow cytometry}

For flow cytometric analysis, the cells were harvested and dissociated into single cells with $0.25 \%$ trypsin and then washed in PBS plus $0.5 \%$ BSA twice. After washing, the cells were stained with antibodies to CXCR4 (FAB170P; R\&D system), c-Kit (FAB332A; R\&D system) and DAZL (ab34139; abcam) for $40 \mathrm{~min}$ at $4^{\circ} \mathrm{C}$. After washing by PBS twice, the cell pellet was re-suspended in $300 \mu \mathrm{l}$ PBS for the final flow cytometric analysis of CXCR4 and c-Kit. Besides, the second antibody (ZF-0511, 1:400, Alexa Fluor 488-conjugated goat anti-rabbit IgG, ZSGB-BIO) was added into DAZL treatment for $40 \mathrm{~min}$ at $4^{\circ} \mathrm{C}$. After that, the cells were washed twice with PBS and re-suspended in $300 \mu \mathrm{l}$ of PBS for the final flow cytometric analysis. As a control for the analysis, the undifferentiated cells in a separate tube were treated with a mouse $\mathrm{IgG}_{1}$ APC-conjugated antibody (IC002A; R\&D system) and a mouse $\mathrm{IgG}_{1}$ isotype control-PE (IC002P; R\&D system) for c-Kit and CXCR4, respectively. For DAZL, the undifferentiated cells were only strained with the second antibody. Finally, cells analysis was performed on the Becton-Dickinson FACS Calibur platform.

\section{Statistical analysis}

Results are expressed as mean \pm Standard error of arithmetic mean (SEM). Statistical significance was determined using a Student's $t$-test and one-way ANOVA. Results were considered significant when $p<0.05$.

\section{CONFLICTS OF INTEREST}

The authors declare no conflicts of interests.

\section{FUNDING}

This work was supported by grants from the National Natural Science Foundation of China (31271605 to Y.P. Sun) and the Independent Innovation Project of Zhengzhou University (13Y10502 to T.T. Cheng). 


\section{Authors' contributions}

T.T.C.: and K.Z.: conception and design, collection and assembly of data, data analysis and interpretation, and manuscript writing; Y.C., G.D.Y., J.H.H., F.W., H.J.K., H.X., H.W.W., M.J., B.G., L.G., Z.G.Y. and Y.Y.W.: content modification; Y.P.S. and G.J.J.: conception and design, data interpretation, manuscript writing, financial support, final approval of the manuscript. All authors reviewed this manuscript.

\section{REFERENCES}

1. Aflatoonian B, Ruban L, Jones M, Aflatoonian R, Fazeli A, Moore HD. In vitro post-meiotic germ cell development from human embryonic stem cells. Human reproduction. 2009; 24:3150-3159.

2. Chen HF, Kuo HC, Chien CL, Shun CT, Yao YL, Ip PL, Chuang CY, Wang CC, Yang YS, Ho HN. Derivation, characterization and differentiation of human embryonic stem cells: comparing serum-containing versus serum-free media and evidence of germ cell differentiation. Human reproduction. 2007; 22:567-577.

3. Clark AT, Bodnar MS, Fox M, Rodriquez RT, Abeyta MJ, Firpo MT, Pera RA. Spontaneous differentiation of germ cells from human embryonic stem cells in vitro. Human molecular genetics. 2004; 13:727-739.

4. Mikkola M, Olsson C, Palgi J, Ustinov J, Palomaki T, HorelliKuitunen N, Knuutila S, Lundin K, Otonkoski T, Tuuri T. Distinct differentiation characteristics of individual human embryonic stem cell lines. BioMed Central. 2006; 6:40.

5. Tilgner K, Atkinson SP, Golebiewska A, Stojkovic M, Lako M, Armstrong L. Isolation of primordial germ cells from differentiating human embryonic stem cells. Stem cells. 2008; 26:3075-3085.

6. West FD, Machacek DW, Boyd NL, Pandiyan K, Robbins KR, Stice SL. Enrichment and differentiation of human germ-like cells mediated by feeder cells and basic fibroblast growth factor signaling. Stem cells. 2008; 26:2768-2776.

7. Bucay N, Yebra M, Cirulli V, Afrikanova I, Kaido T, Hayek A, Montgomery AM. A novel approach for the derivation of putative primordial germ cells and sertoli cells from human embryonic stem cells. Stem cells. 2009; 27:68-77.

8. Geens M, Sermon KD, Van de Velde H, Tournaye H. Sertoli cell-conditioned medium induces germ cell differentiation in human embryonic stem cells. Journal of assisted reproduction and genetics. 2011; 28:471-480.

9. Kee K, Angeles VT, Flores M, Nguyen HN, Reijo Pera RA. Human DAZL, DAZ, BOULE genes modulate primordial germ-cell and haploid gamete formation. Nature. 2009; 462:222-225.

10. Lim JJ, Shim MS, Lee JE, Lee DR. Three-step method for proliferation and differentiation of human embryonic stem cell (hESC)-derived male germ cells. PloS one. 2014; 9:e90454.

11. Medrano JV, Ramathal C, Nguyen HN, Simon C, Reijo Pera RA. Divergent RNA-binding proteins, DAZL, VASA, induce meiotic progression in human germ cells derived in vitro. Stem cells. 2012; 30:441-451.

12. KEHKOOI KEE JMG, AMANDER T. CLARK, and PERA aRAR. Bone Morphogenetic Proteins Induce Germ Cell Differentiation from Human Embryonic Stem Cells. Stem cells and development. 2006; 15:831-837.

13. Easley CAt, Phillips BT, McGuire MM, Barringer JM, Valli H, Hermann BP, Simerly CR, Rajkovic A, Miki T, Orwig KE, Schatten GP. Direct differentiation of human pluripotent stem cells into haploid spermatogenic cells. Cell reports. 2012; 2:440-446.

14. Park TS, Galic Z, Conway AE, Lindgren A, van Handel BJ, Magnusson M, Richter L, Teitell MA, Mikkola HK, Lowry WE, Plath K, Clark AT. Derivation of primordial germ cells from human embryonic and induced pluripotent stem cells is significantly improved by coculture with human fetal gonadal cells. Stem cells. 2009; 27:783-795.

15. Liu T, Huang Y, Liu J, Zhao Y, Jiang L, Huang Q, Cheng W, Guo L. MicroRNA-122 influences the development of sperm abnormalities from human induced pluripotent stem cells by regulating TNP2 expression. Stem cells and development. 2013; 22:1839-1850.

16. Eguizabal C, Montserrat N, Vassena R, Barragan M, Garreta E, Garcia-Quevedo L, Vidal F, Giorgetti A, Veiga A, Izpisua Belmonte JC. Complete meiosis from human induced pluripotent stem cells. Stem cells. 2011; 29:1186-1195.

17. Sasaki K, Yokobayashi S, Nakamura T, Okamoto I, Yabuta Y, Kurimoto K, Ohta H, Moritoki Y, Iwatani C, Tsuchiya H, Nakamura S, Sekiguchi K, Sakuma T, et al. Robust In Vitro Induction of Human Germ Cell Fate from Pluripotent Stem Cells. Cell stem cell. 2015; 17:178-194.

18. West FD, Roche-Rios MI, Abraham S, Rao RR, Natrajan MS, Bacanamwo M and Stice SL. KIT ligand and bone morphogenetic protein signaling enhances human embryonic stem cell to germ-like cell differentiation. Human reproduction. 2010; 25:168-178.

19. Nayernia K, Nolte J, Michelmann HW, Lee JH, Rathsack K, Drusenheimer N, Dev A, Wulf G, Ehrmann IE, Elliott DJ, Okpanyi V, Zechner U, Haaf T, et al. In vitro-differentiated embryonic stem cells give rise to male gametes that can generate offspring mice. Developmental cell. 2006; 11:125-132.

20. Hayashi K, Ohta H, Kurimoto K, Aramaki S, Saitou M. Reconstitution of the mouse germ cell specification pathway in culture by pluripotent stem cells. Cell. 2011; 146:519-532.

21. Mark M, Teletin M, Vernet N, Ghyselinck NB. Role of retinoic acid receptor (RAR) signaling in post-natal male germ cell differentiation. Biochimica et biophysica acta. 2015; 1849:84-93.

22. Endo T, Romer KA, Anderson EL, Baltus AE, de Rooij DG, Page DC. Periodic retinoic acid-STRA8 signaling 
intersects with periodic germ-cell competencies to regulate spermatogenesis. Proceedings of the National Academy of Sciences of the United States of America. 2015; 112:E2347-2356.

23. Kimura $T$, Nakamura $T$, Murayama $K$, Umehara $H$, Yamano N, Watanabe S, Taketo MM, Nakano T. The stabilization of beta-catenin leads to impaired primordial germ cell development via aberrant cell cycle progression. Developmental biology. 2006; 300:545-553.

24. Lombardi AP, Royer C, Pisolato R, Cavalcanti FN, Lucas TF, Lazari MF, Porto CS. Physiopathological aspects of the Wnt/beta-catenin signaling pathway in the male reproductive system. Spermatogenesis. 2013; 3:e23181.

25. Nicol B, Guiguen Y. Expression profiling of Wnt signaling genes during gonadal differentiation and gametogenesis in rainbow trout. Sexual development : 2011; 5:318-329.

26. Tanwar PS, Kaneko-Tarui T, Zhang L, Rani P, Taketo MM, Teixeira J. Constitutive WNT/beta-catenin signaling in murine Sertoli cells disrupts their differentiation and ability to support spermatogenesis. Biology of reproduction. 2010; 82:422-432.

27. Clevers H, Nusse R. Wnt/beta-catenin signaling and disease. Cell. 2012; 149:1192-1205.

28. Angers S, Moon RT. Proximal events in Wnt signal transduction. Nature reviews Molecular cell biology. 2009; $10: 468-477$.

29. Nelson WJ, Nusse R. Convergence of Wnt, beta-catenin, and cadherin pathways. Science. 2004; 303:1483-1487.

30. Busada JT, Chappell VA, Niedenberger BA, Kaye EP, Keiper BD, Hogarth CA, Geyer CB. Retinoic acid regulates Kit translation during spermatogonial differentiation in the mouse. Developmental biology. 2015; 397:140-149.

31. Molyneaux KA. The chemokine SDF1/CXCL12 and its receptor CXCR4 regulate mouse germ cell migration and survival. Development. 2003; 130:4279-4286.

32. fishKee K, Angeles VT, Flores M, Nguyen HN, Reijo Pera RA. Human DAZL, DAZ, BOULE genes modulate primordial germ-cell and haploid gamete formation. Nature. 2009; 462:222-225.

33. Heyting C. Synaptonemal complexes: structure and function. Current opinion in cell biology. 1996; 8:389-396.

34. Huang TS, Li L, Moalim-Nour L, Jia D, Bai J, Yao Z, Bennett SA, Figeys D, Wang L. A Regulatory Network Involving beta-Catenin, e-Cadherin, PI3k/Akt, and Slug Balances Self-Renewal and Differentiation of Human Pluripotent Stem Cells In Response to Wnt Signaling. Stem cells. 2015; 33:1419-1433.

35. Chen B, Dodge ME, Tang W, Lu J, Ma Z, Fan CW, Wei S, Hao W, Kilgore J, Williams NS, Roth MG, Amatruda JF, Chen C, et al. Small molecule-mediated disruption of Wntdependent signaling in tissue regeneration and cancer. Nature chemical biology. 2009; 5:100-107.

36. Chen HH, Welling M, Bloch DB, Munoz J, Mientjes E, Chen X, Tramp C, Wu J, Yabuuchi A, Chou YF, Buecker C,
Krainer A, Willemsen R, et al. DAZL limits pluripotency, differentiation, and apoptosis in developing primordial germ cells. Stem cell reports. 2014; 3:892-904.

37. Yu Z, Ji P, Cao J, Zhu S, Li Y, Zheng L, Chen X, Feng L. Dazl promotes germ cell differentiation from embryonic stem cells. Journal of molecular cell biology. 2009; 1:93-103.

38. Chung SS, Wolgemuth DJ. Role of retinoid signaling in the regulation of spermatogenesis. Cytogenetic and genome research. 2004; 105:189-202.

39. Dann CT, Alvarado AL, Molyneux LA, Denard BS, Garbers DL, Porteus MH. Spermatogonial stem cell selfrenewal requires OCT4, a factor downregulated during retinoic acid-induced differentiation. Stem cells. 2008; 26:2928-2937.

40. Wan Q, Lu H, Wu LT, Liu X, Xiang JB. Retinoic acid can induce mouse embryonic stem cell R1/E to differentiate toward female germ cells while oleanolic acid can induce $\mathrm{R} 1 / \mathrm{E}$ to differentiate toward both types of germ cells. Cell biology international. 2014; 38:1423-1429.

41. Chen HF, Jan PS, Kuo HC, Wu FC, Lan CW, Huang MC, Chien CL, Ho HN. Granulosa cells and retinoic acid cotreatment enrich potential germ cells from manually selected Oct4-EGFP expressing human embryonic stem cells. Reproductive biomedicine online. 2014; 29:319-332.

42. Li P, Hu H, Yang S, Tian R, Zhang Z, Zhang W, Ma M, Zhu Y, Guo X, Huang Y, He Z, Li Z. Differentiation of induced pluripotent stem cells into male germ cells in vitro through embryoid body formation and retinoic acid or testosterone induction. BioMed research international. 2013; 2013:608728.

43. Silva C, Wood JR, Salvador L, Zhang Z, Kostetskii I, Williams CJ, Strauss JF, 3rd. Expression profile of male germ cell-associated genes in mouse embryonic stem cell cultures treated with all-trans retinoic acid and testosterone. Molecular reproduction and development. 2009; 76:11-21.

44. Lam AQ, Freedman BS, Morizane R, Lerou PH, Valerius MT, Bonventre JV. Rapid and efficient differentiation of human pluripotent stem cells into intermediate mesoderm that forms tubules expressing kidney proximal tubular markers. Journal of the American Society of Nephrology. 2014; 25:1211-1225.

45. Araoka $\mathrm{T}$, Mae S, Kurose $\mathrm{Y}$, Uesugi M, Ohta A, Yamanaka S, Osafune K. Efficient and rapid induction of human iPSCs/ESCs into nephrogenic intermediate mesoderm using small molecule-based differentiation methods. PloS one. 2014; 9:e84881.

46. Aguilar JS, Begum AN, Alvarez J, Zhang XB, Hong Y, Hao J. Directed cardiomyogenesis of human pluripotent stem cells by modulating Wnt/beta-catenin and BMP signalling with small molecules. The Biochemical journal. 2015; 469:235-241.

47. Lian X, Zhang J, Azarin SM, Zhu K, Hazeltine LB, Bao X, Hsiao C, Kamp TJ, Palecek SP. Directed cardiomyocyte differentiation from human pluripotent stem cells by 
modulating Wnt/beta-catenin signaling under fully defined conditions. Nature protocols. 2013; 8:162-175.

48. Wu Y, Liu F, Liu Y, Liu X, Ai Z, Guo Z, Zhang Y. GSK3 inhibitors CHIR99021 and 6-bromoindirubin-3'-oxime inhibit microRNA maturation in mouse embryonic stem cells. Scientific reports. 2015; 5:8666.
49. Wang F, Kong HJ, Kan QC, Liang JY, Zhao F, Bai AH, Li PF, Sun YP. Analysis of blastocyst culture of discarded embryos and its significance for establishing human embryonic stem cell lines. Journal of cellular biochemistry. 2012; 113:3835-3842. 